\title{
474370 - ANESTHESIA FOR TRANS-CATHETER AORTIC VALVE IMPLANTATION: A CASE SERIES
}

\author{
Massimiliano Meineri, MD, Marcin Wasowicz, MD, PHD, Annette Vegas, MD, \\ FRCPC, Jacek Karski, MD, FRCPC \\ Department of Anesthesia and Pain Management, Toronto General Hospital, \\ Toronto, ON, Canada
}

Purpose: Trans-catheter Aortic Valve Implantation (T-C AVR) has been offered, on a compassionate basis, to patients with symptomatic aortic valve (AV) stenosis deemed to be too high risk for surgery[1,2]. The procedure consists of two steps: an AV balloon valvuloplasty, followed by the deployment of a pericardial bioprosthesis. Both steps are carried out using rapid ventricular pacing to suppress left ventricular ejection. The native AV can be approached antegrade through the LV apex via a small thoracotomy [2] or retrograde through percutaneous access to the femoral artery[1]. General anesthesia is the standard of practice.

Clinical Features: Local REB approval was obtained for this study. From January to December 2007, 14 patients underwent T-C AVR at our institution. Patients' characteristics and outcomes are described in table 1. The Edwards SAPIEN transcatheter aortic valve was used in all cases. The trans-femoral approach was performed by an interventional cardiologist in the cardiology suite. General anesthesia with a single lumen endotracheal intubation, large bore peripheral intravenous line, arterial line and double lumen central venous line was used. A temporary trans-venous wire was inserted for pacing. A fluid warmer and forced air warming blanket were used. After deployment of the valve patients were transferred to the operating room (OR) for surgical removal of the $24 \mathrm{~F}$ femoral sheath and repair of femoral artery. 4 patient were extubated in the OR, 2 patients within ours in the coronary care unit. The trans-apical approach was performed in the cardiovascular OR by a cardiac surgeon under standard cardiac anesthesia. The Swan-Ganz catheter was advanced after valve deployment. Temporary epicardial wires were inserted for pacing. A continuous flow cell-saver system was used. After transfer to the cardiovascular intensive care unit, mechanical ventilation was weaned as per institutional protocols.

Conclusion: T-C AVR, is a viable option in a subset of patients not previously considered for surgery. All patients can potentially need rescue open surgery at any time during the procedure. Expertise in cardiac anesthesia and knowledge of all technical aspects of the procedure is thus mandatory.

References: [1] Circulation 2007;116:755-763. [2] Circulation 2007;116[suppl]:I-240-I245.

TAB. 1 PATIENTS CHARACTERISTICS AND OUTCOME 


\begin{tabular}{|c|c|c|}
\hline Approach & Trans-femoral & Tras-apical \\
\hline \multicolumn{2}{|c|}{ Baseline Characteristics } \\
\hline Number & 7 & 7 \\
\hline Age y mean (range) & $83.7(68-93)$ & $78.7(73-81)$ \\
\hline Female gender & $2(28)$ & $6(86)$ \\
\hline Body Mass Index mean (range) & $1.76(1.6-2.10)$ & $1.6(1.42-1.85)$ \\
\hline Previous CV surgery & $3(42)$ & $3(42)$ \\
\hline Severe COPD & 0 & $5(71)$ \\
\hline CAD & $6(86)$ & $1(57)$ \\
\hline LV Grade 3-4 & 0 & 20.45 \\
\hline Re-thoracotomy for bleeding & 19.69 & $1(14)$ \\
\hline Predicted 30 days mortality* mean & 0 & $1(14)$ \\
\hline Deriprocedural Stroke & 0 & 0 \\
\hline Valve embolization & 0 & $1(14)$ \\
\hline Conversion to open procedure & 0 & $1(28)$ \\
\hline RBC transfused $>2$ U & 0 & $14)$ \\
\hline
\end{tabular}

Values are expressed as n (\%)unless otherwise specified. *Logistic EuroSCORE 